\title{
Evaluation of surgical capabilities is complex but necessary
}

\section{Andrea J. Carpenter, MD, PhD}

Surgical capabilities are complex including technical ability, patient management, and judgement. The evaluation of surgical capability is very challenging, but is imperative to assure the excellent care our patients expect and deserve. Technical skills include the ability to accurately identify anatomy and pathology, to handle tissue, to cut, sew, tie knots, and manipulate scopes or robots. These skills can be very complex and challenging to assess. However, when complex skills are broken down to fundamental elements, there are tools available for evaluation, ${ }^{1}$ but really assessing the ability to combine skills efficiently remains difficult. $^{2}$

Patient management includes preparation and operative planning, postoperative care, and dealing with inevitable complications. Written and oral examination can reasonably test patient management skills at least for more common situations, but full capability must include clinical judgement. Judgement begins with knowledge of the specific pathology to be treated, evolves with experience, and changes over time as numerous factors influence cognitive ability such as environmental stressors and aging. The bottom line is that our society expects that we are capable in all of these areas and believes that we monitor our ranks to assure that is true.

It is expected that all surgeons are capable, but are all capabilities equal? There have been numerous reports that have compared surgical outcomes on the basis of surgeon specialty and experience. ${ }^{3,4}$ General thoracic and cardiac surgeons have historically had the same training but differ by experience with the thoracic surgeon performing lung cases as a larger part of their practice. General surgeons begin with different training, but might perform as many, or more, lung cases as the thoracic surgeon. In this large national sample, ${ }^{4}$ there are clear mortality differences with the specialty trained and experienced thoracic surgeon having

From the Department of Cardiothoracic Surgery, Joe R. and Teresa Lozano Long School of Medicine, San Antonio, Tex.

Presented at the 101st Annual Meeting of The American Association for Thoracic Surgery, Cardiothoracic Ethics Forum: Measuring surgical competence: Why, when, how, and what to do about it. May 2, 2021.

Received for publication Sept 27, 2021; revisions received Dec 8, 2021; accepted for publication Jan 10, 2022; available ahead of print Jan 20, 2022.

Address for reprints: Andrea J. Carpenter, MD, PhD, Department of Cardiothoracic Surgery, 7703 Floyd Curl Dr, MC 7841, San Antonio, TX 78229 (E-mail: Carpentera2@uthscsa.edu).

J Thorac Cardiovasc Surg 2022;164:1014-6

$0022-5223 / \$ 36.00$

Copyright $($ c 2022 by The American Association for Thoracic Surgery

https://doi.org/10.1016/j.jtcvs.2022.01.015

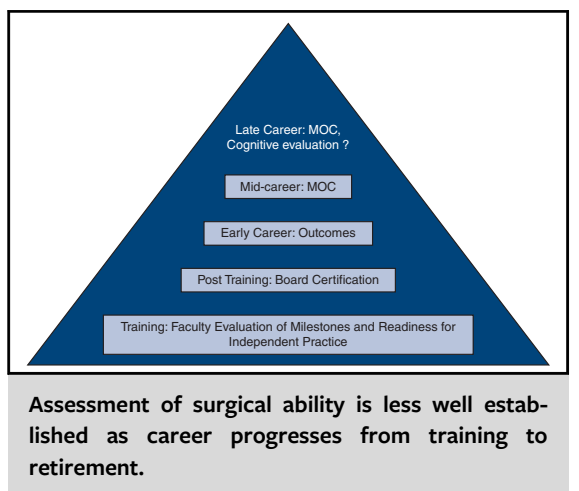

CENTRAL MESSAGE

Our society expects and deserves assurance that every surgeon can provide excellent care. It is incumbent on our profession to assure this is true, but current assessment methods are inadequate.

See Commentary on page 1017

the lowest mortality compared with either cardiac or general surgeons.

Just as surgeon capability varies by training and experience, so capability varies over time for each surgeon. Early in our careers, training program faculty judge the capability of the surgeon and declare them ready for independent practice at the conclusion of a usually predetermined time. There is inevitably variability among faculty evaluators as to an individual trainee's capability. Over the past 2 decades there has been a growing interest in competency-based training: simulation is evolving as an educational tool and a method to evaluate technical skill. ${ }^{1}$ More recently the concept of defining entrustable professional activities that can be specialty-, disease-, or procedure-specific is gaining popularity as an assessment tool, although still very immature. ${ }^{5,6}$ Outcomes databases such as the Society of Thoracic Surgeons, Northern New England Cardiovascular Disease Study Group, Virginia Cardiac Services Quality Initiative, and Michigan Society of Thoracic and Cardiovascular Surgeons Quality Collaborative databases are gradually moving toward comparisons of individual surgeons with 


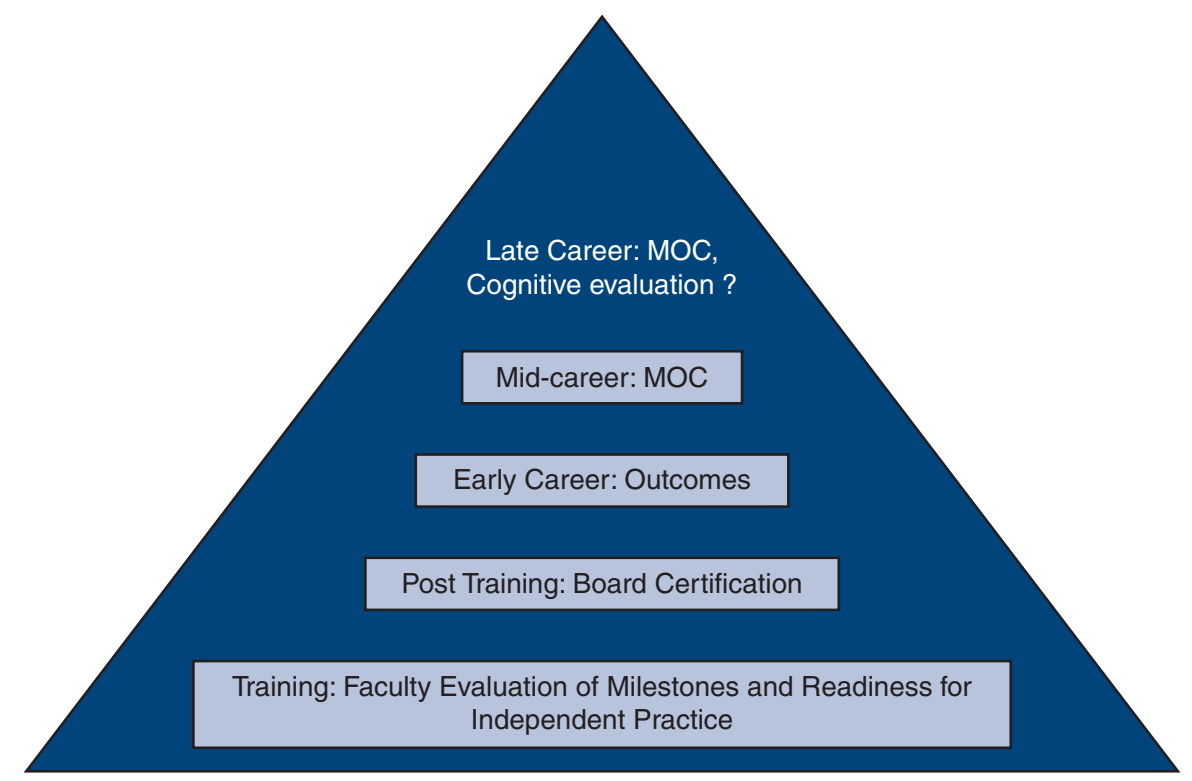

FIGURE 1. Over the course of a surgeon's career the need to assess ability becomes progressively complex because of changing dexterity, endurance, and cognitive function associated with experience and aging. The available tools to make these assessments are few and poorly validated. $M O C$, Maintenance of Certification.

national or regional cohorts as an objective assessment of capability. At present the validity and reliability of competency-based assessments are still early and unproven but moving forward none the less.

The very foundation of clinical judgement must begin with knowledge of disease processes, diagnostic testing, and therapeutic options. We all understand that judgement matures with experience, but we do not yet understand how to evaluate the point when judgement is "good" or at least "good enough." We do not know when experience means judgement is optimal. We suspect that judgement and ability decline with age, but we have few reliable means of measuring that decline or deciding when surgical ability is no longer "good" or at least "good enough." It is also difficult to assess when judgement and skill mature to a peak, and we should not undervalue the benefit of a senior surgeon's effect on the development of a junior surgeon. Senior surgeons should consider the value of mentorship to the newer members of the group when planning for retirement.

We know that individuals age at different rates and aging affects physical and mental stamina, coordination, reaction time, and judgement. The Cognitive Changes and Retirement among Senior Surgeons Study (CCRSS) was conducted during the American College of Surgeons Clinical Congress over a 6-year period. $^{7-9}$ Computerized cognitive tasks measuring sustained attention, reaction time, visual learning, and memory were administered to 168 senior surgeons aged 60 years or older and compared with data from 126 younger surgeons aged 45 to 59 years. Several reports from these studies have been published. These reports make for interesting reading and reflection, and the fundamental findings can be summarized as follows:

- Most practicing senior surgeons performed at or near the level of their younger peers on all cognitive tasks, as did almost half of the retired senior surgeons.

- There was no notable relationship between subjective cognitive change and objective measures of cognitive function.

- Self-perceived cognitive change influenced retirement decisions. Surgeons chose to retire for concern of decline in cognitive ability although objective testing showed preservation of cognitive ability, possibly related to the continued mental stimulation of their careers.

- The CCRSS studies show that surgeon decisions to retire are influenced by subjective concerns of declining cognitive ability whereas objective measures do not match the subjective concerns.

The medical executive staff at Yale New Haven Hospital reported the results of a locally developed screening battery intended to assess cognitive function among senior staff requesting to recredential. ${ }^{10}$ The authors reported on screening of 141 providers, including physicians, dentists, psychologists, podiatrists, and midlevel providers. After screening, 115 proceeded to recredential with planned repeat screening in 1 to 2 years. Twenty-six had further evaluation with 8 proceeding to recredential. The remaining 18 entered proctored practice, retired, or resigned. In this report, the 2 most important steps in this assessment process are the selection of a neuropsychologist and members of the 
Review Committee to evaluate the results. They concluded that cognitive testing applied by a skilled neuropsychologist is likely an effective approach to identifying individuals with impaired cognitive skills that could affect their ability to practice medicine. However, there is yet no clearly validated method for cognitive testing that might be widely distributed.

Consider the need to evaluate surgical ability in view of the demand for surgical care. The Association of American Medical Colleges 2021 update report on the physician workforce $^{11}$ projected shortages in all specialties over the next 15 years, with surgical specialty demand exceeding supply by 15,800 to 30,200 surgeons by the year 2034 . At present there are approximately 3640 cardiothoracic surgeons practicing the United States, and $18 \%$ of these surgeons are 65 years or older. During the late 1990s the number of applicants to cardiothoracic residency declined resulting in program closures and fewer graduates entering the workforce. The result is an increased reliance on older surgeons to maintain an adequate workforce. ${ }^{11}$ The CCRSS study showed premature retirements because of concern for declining cognitive ability that was not supported by objective cognitive testing. The profession could benefit when senior surgeons plan for retirement while assuring skilled replacements through mentorship and seeking objective cognitive testing if concerned about their own ability.

To summarize the evaluation of surgical ability is a complex but necessary process. Society has a reasonable expectation to receive safe and effective surgical care. At present, we have imperfect systems in place to accomplish objective evaluation of all aspects of surgical practice. As a surgical career progresses, we have more complex requirements but fewer validated tools for assessment (Figure 1), to include changes in dexterity and cognitive function with age. We should work to develop evaluation methodology that is reliable enough to make assessment of surgical abilities mandatory from medical school graduation to retirement. Only through such effort can we sustain an adequate workforce by graduating able surgeons, limiting premature retirements to assure competent mentorship during early practice, and protecting against declining competence with age.

\section{Conflict of Interest Statement}

The author reported no conflicts of interest.

The Journal policy requires editors and reviewers to disclose conflicts of interest and to decline handling or reviewing manuscripts for which they may have a conflict of interest. The editors and reviewers of this article have no conflicts of interest.

\section{References}

1. Scott DJ, Valentine RJ, Bergen PC, Rege RV, Laycock R, Tesfay ST, et al. Evaluating surgical competency with the American Board of Surgery In-Training Examination, skill testing, and intraoperative assessment. Surgery. 2000;128:613-22.

2. Williams RG, George BC, Bohnen JD, Dunnington GL, Fryer JP, Klamen DL, et al. A proposed blueprint for operative performance training, assessment, and certification. Ann Surg. 2021;273:701-8.

3. Silvestri GA, Handy J, Lackland D, Corley E, Reed CE. Specialists achieve better outcomes than generalists for lung cancer surgery. Chest. 1998;114:675-80.

4. Schipper PH, Diggs BS, Ungerleider RM, Welke KF. The influence of surgeon specialty on outcomes in general thoracic surgery: a national sample 1996 to 2005. Ann Thorac Surg. 2009;88:1566-72; discussion: 1572-3.

5. Brasel KJ, Klingensmith ME, Englander R, Grambau M, Buyske J, Sarosi G, et al. Entrustable Professional activities in general surgery: development and implementation. J Surg Educ. 2019;76:1174-86.

6. Stahl CC, Collins E, Jung SA, Rosser AA, Kraut AS, Schnapp BH, et al. Implementation of entrustable professional activities into a general surgery residency. $J$ Surg Educ. 2020;77:739-48.

7. Drag LL, Bieliauskas LA, Langenecker SA, Greenfield LJ. Cognitive functioning, retirement status, and age: results from the cognitive changes and retirement among senior surgeons study. J Am Coll Surg. 2010;211:303-7.

8. Lee HJ, Drag LL, Bieliauskas LA, Langenecker SA, Graver C, O'Neill J, et al. Results from the cognitive changes and retirement among senior surgeons selfreport survey. J Am Coll Surg. 2009;209:668-71.e2.

9. Bieliauskas LA, Langenecker S, Graver C, Lee HJ, O'Neill J, Greenfield LJ. Cognitive changes and retirement among senior surgeons (CCRASS): results from the CCRASS study. J Am Coll Surg. 2008;207:69-78; discussion: 78-9. https://doi.org/10.1016/j.jamcollsurg.2008.01.022

10. Cooney L, Balcezak T. Cognitive testing of older clinicians prior to recredentialing. JAMA. 2020;323:179-80.

11. AAMC. Physician workforce projections. The complexities of physician supply and demand: projections from 2019 to 2034. Accessed August 12, 2021. https:// www.aamc.org/data-reports/workforce/data/complexities-physician-supply-anddemand-projections-2019-2034

Key Words: competency assessment, surgical judgement, technical skill, cognitive function, clinical performance, aging 